\title{
Best Practices: Improving Patient Outcomes and Costs in an ACO Through Comprehensive Medication Therapy Management
}

\author{
Amanda Brummel, PharmD, BCACP; Adam Lustig, MS; Kimberly Westrich, MA;
}

Michael A. Evans, RPh; Gary S. Plank, PharmD; Jerry Penso, MD, MBA; and Robert W. Dubois, MD, PhD

\begin{abstract}
BACKGROUND: One of the most important and often overlooked challenges for accountable care organizations (ACOs) is ensuring the optimal use of pharmaceuticals, which can be accomplished by utilizing pharmacists' skillsets and leveraging their full clinical expertise. Developing capabilities that support, monitor, and ensure appropriate medication use, efficacy, and safety is critical to achieving optimal patient outcomes and, ultimately, to an ACO's success. The program described in this article highlights the best practices of Fairview Pharmacy Services' Medication Therapy Management (MTM) program with additional thoughts and considerations on this and similar MTM programs provided by The Working Group on Optimizing Medication Therapy in Value-Based Healthcare.

PROGRAM DESCRIPTION: Fairview Pharmacy Services utilizes 23 MTM pharmacists (approximately 18 full-time equivalents) working in 30 locations, who conduct pharmacotherapy workups as part of the MTM services that Fairview provides. Pharmacists focus on patients in a comprehensive manner and assess all of their diseases and medications. Responsibilities include (a) identification of a patient's drug-related needs with a commitment to meet those needs; (b) an assessment and confirmation that all of a patient's drug therapy is appropriately indicated, effective and safe, and that the patient is compliant; (c) achievement of therapy outcomes and ensuring documentation of those outcomes; and (d) collaboration with all members of a patient's care team.
\end{abstract}

OBSERVATIONS: Since 1998, pharmacists have cared for more than 20,000 patients and resolved more than 107,000 medication-related problems which, if left unresolved, could have led to hospital readmissions and emergency visits. Since becoming a Pioneer ACO, Fairview pharmacists have focused on the highest-risk members and have seen over 670 ACO patients, resolving over 2,780 medication-related problems. In terms of clinical outcomes, MTM contributed to optimal care in complex patients with diabetes. A review of 2007 data found that the percentage of diabetes patients optimally managed (as measured by a composite of hemoglobin A1c, low-density lipoprotein, blood pressure, aspirin use, and no smoking) was significantly higher for MTM patients $(21 \%$ vs. $45 \%, P<0.01)$. The Fairview MTM also showed a 12:1 return on investment (ROI) when comparing the overall health care costs of patients receiving MTM services with patients who did not receive those services.

IMPLICATIONS: Developing an MTM program to manage and optimize pharmaceuticals will be a cornerstone to managing the health of a population. Important lessons have been learned that may be helpful to other health systems developing MTM programs. In an accountable care environment measuring the return on the investment of all care interventions, including MTM will be essential to maintain the program. The ACO will also have to be able to correctly identify which patients are candidates for MTM services and provide pharmacists with enough autonomy, including scheduling face-to-face interactions with patients and the ability to change prescriptions if necessary, to ensure that timely and effective care is delivered. In order for an ACO to deliver high quality patient-centered medication services, there must be clear lines of communication between providers, pharmacists, and the other care providers within the organization. Finally, a strong and visionary leader is critical to ensuring the success of an MTM program and ultimately the ACO itself.

RECOMMENDATIONS: While there is a plethora of literature touting the benefits of either in-person or telephonic-based MTM, there is little research to date that directly compares these 2 MTM delivery types. It is critical for research to address the direct and indirect costs associated with starting and maintaining an MTM program. Information such as technologies required to start a program and length of time until a program breaks even or meets a sufficient ROI can be helpful for health care providers in similar health systems pitching a similar type of program. Finally, there has yet to be significant empirical research into the cost savings of utilizing a pharmacist and MTM services associated with meeting quality and cost benchmarks in an accountable care payment arrangement.

\section{J Manag Care Pharm. 2014;20(12):1152-58}

Copyright $\odot 2014$, Academy of Managed Care Pharmacy. All rights reserved.

\section{What is already known about this subject}

Pharmacists play a critical role in delivering high-value health care and can successfully be integrated into a care team. Medication therapy management (MTM) programs have been shown to be effective in managing high-risk populations MTM programs have also been shown to lower total health expenditures

\section{What this study adds}

MTM programs can play a critical role in an accountable care organization (ACO) meeting its quality and financial benchmarks.

Details and preliminary data on a comprehensive MTM program show how an already established MTM program can transition to address the needs of a high-risk ACO population.

T aking care of patients in risk-based models such as accountable care organizations (ACOs) rather than the traditional fee-for-service model presents health care providers with new challenges, some of which they are readily prepared to meet and others for which they must adopt proven solutions or develop new ones. With more than 20 million patients now receiving care from 1 of the 493 ACOs in existence, it is critical that providers participating in these new care models are provided the resources and knowledge necessary 
to overcome these new challenges. ${ }^{1}$ One of the most important and often overlooked challenges for ACOs is ensuring the optimal use of pharmaceuticals, which can be accomplished by utilizing pharmacists' skillsets and leveraging their full clinical expertise. ${ }^{2}$ With some ACOs struggling to effectively manage medication-related capabilities, organizations should look for effective ways to leverage pharmacists as an integral part of patient care. One such way to take advantage of pharmacist skill and expertise is through medication therapy management (MTM) programs. ${ }^{3}$

Developing capabilities that support, monitor, and ensure appropriate medication use, efficacy, and safety is critical to achieving optimal patient outcomes and, ultimately, to an ACO's success. MTM is defined by the American Pharmacists Association as a service or group of services that optimizes therapeutic outcomes for individual patients and may include medication therapy reviews, pharmacotherapy consults, anticoagulation management, and many other clinical services. ${ }^{4}$ Previous research has shown that pharmacist-led MTM can reduce costs, improve patient care, and reduce physician workload. $^{5-10}$ Some health care systems are further along in implementing, evaluating, and improving these types of services than others. While many ACOs approach care delivery in different ways, they are all focused on quality and financial benchmarks and therefore have the potential to learn from each other. The experiences and solutions of more advanced ACOs may help other organizations achieve success more quickly. To that end, this article highlights the best practices of Fairview Pharmacy Services' MTM program, which focuses on conducting a full pharmacotherapy work-up and setting goals for patients with chronic health issues to improve patient outcomes and reduce costs.

\section{Program Description}

Fairview Pharmacy Services' MTM program, developed in partnership with the University of Minnesota College of Pharmacy, has been helping people manage chronic health conditions and maximize the therapeutic value of medicines for more than 15 years. The Fairview MTM program discussed in this case study is not to be confused with the MTM program required by the Medicare Part D program. The aims of the programs are similar, but the Part D MTM program is available only to certain Medicare recipients and is not the comprehensive approach that Fairview has developed. Based on the pharmaceutical care philosophy, Fairview's MTM program was established prior to the MTM concept included in the Medicare Prescription Drug, Improvement and Modernization Act of 2003, which requires an MTM program for eligible beneficiaries. ${ }^{6}$

The Fairview MTM was established in 1997 to assist the health system in the management of medications and to train pharmacy students. The goals were to provide comprehensive medication management services, identify and resolve drug therapy problems, and help maximize drug therapy while reducing adverse drug events and producing savings. The target population was those patients on multiple medications with chronic conditions who were not meeting their therapy goals.

During the past several years, the MTM program has evolved as the Minneapolis-based Fairview Health System has participated in the Centers for Medicare and Medicaid Services (CMS) Pioneer ACO model and has initiated shared-saving contracts with several commercial payers. CMS defines an ACO as groups of doctors, hospitals, and other health care providers, who come together voluntarily to give coordinated high quality care to their Medicare patients. ${ }^{11}$ When contracting as an ACO, a system or entity will likely participate in an agreement that includes the possibility of shared savings or, as in the Pioneer ACO, where there can be an up or downside incentive depending on the quality and cost results of the ACO.

Fairview Health Services, in partnership with the University of Minnesota, is a network of 7 hospitals, 44 primary care clinics, 55 specialty clinics, and 30-plus retail pharmacies that serves the greater Minneapolis-St. Paul area as well as communities throughout Minnesota and the upper Midwest. The not-forprofit corporation is one of the largest health care providers in Minnesota. There are now 23 MTM pharmacists (approximately 18 full-time equivalents) working in 30 locations-typically in primary care/internal medicine clinics, but some are in specialty sites. Fairview has developed the Fairview Health Network that includes employed providers and also independent providers affiliated with Fairview Physician Associates. Fairview has care team capabilities including MTM pharmacists, care coordination, diabetes educators, health coaches, and behavioral health clinicians that assist in population management. The Fairview MTM program is a model other health care systems can emulate as they develop the resources and programs to successfully navigate the emerging $\mathrm{ACO}$ environment.

Each MTM encounter involves a pharmacotherapy workup - a systematic review designed to identify and resolve drug therapy problems and ensure optimal patient outcomes. To aid in this review, 3 forms are used: a detailed assessment form, a form for a care plan, and an evaluation form. ${ }^{12}$ The pharmacist focuses on the patient in a comprehensive manner and assesses all of the patient's diseases and medications. Pharmacist responsibilities include (a) identification of a patient's drug-related needs with a commitment to meet those needs; (b) an assessment and confirmation that all of a patient's drug therapy is appropriately indicated, effective and safe, and that the patient is compliant; (c) achievement of therapy outcomes and ensuring documentation of those outcomes; and (d) collaboration with all members of a patient's care team.

Therapeutic goals are established for each of a patient's medical conditions during the care plan development. The patient, prescriber, and pharmacist communicate either face-to-face at the clinic, electronically via the electronic medical record 
(EMR), or telephonically to discuss patient expectations and goals of therapy. For a number of medical conditions, such as diabetes, there are collaborative practice agreements in place so the MTM pharmacist can initiate, modify, or discontinue drug therapy as well as order lab tests related to the patient's medical condition, according to established protocols. There is also an overarching protocol in place in addition to condition-specific protocols. Minnesota's pharmacy practice act was modified in 1999 to allow pharmacists to participate in the practice of managing and modifying drug therapy. Each state has its own laws that will determine how pharmacists can participate in MTM programs in a particular state. ${ }^{13}$

Most of the MTM pharmacists are doctors of pharmacy or have residency training. All practitioners must go through a training program offered in conjunction with the University of Minnesota College of Pharmacy. The program ensures that all pharmacists are educated on the comprehensive medication management model, which is consistent with the pharmaceutical care model defined in Pharmaceutical Care Practice: The Patient-Centered Approach to Medication, a definitive resource that provides information on maintaining medication management services, so that a standard approach is used when providing direct patient care. The training program requires practitioners to workup over 50 patients and have these cases reviewed by a pharmaceutical care pharmacist, who is approved by the University of Minnesota College of Pharmacy and who is authorized to review and verify patient encounter documentation.

Fairview MTM pharmacists generally meet with patients in person. This can be done alone or in conjunction with a provider appointment. While some consultations are carried out telephonically and by webcam through a virtual platform vendor (EmergeMD), patients visiting in person seem to build relationships with the MTM pharmacist faster. ${ }^{14}$ The MTM program relies on efficient communication and a team-based approach where the pharmacist is actively seen as a member of the care team.

\section{MTM in an Evolving Delivery and Payment Environment}

A significant change in the MTM program is payment for services. When the program began, pharmacists could not bill for their services. In 2005, the Minnesota legislature authorized coverage of MTM services for medical assistance and general assistance medical care recipients in the state..$^{15}$ The MTM services are covered for recipients "taking three or more prescriptions to treat or prevent one or more chronic medical conditions." In 2006, the Medicare Part D benefit went into effect, and locally, some plans began to directly reimburse for MTM services as well. Fairview also went directly to employers and contracted with them to provide MTM services. In the feefor-service structure, Fairview uses the specified MTM Current Procedural Terminology (CPT) codes 99605, 99606, and
99607. The MTM payment may be based on time spent with each patient, following the previously mentioned CPT codes, or based on patient complexity (e.g., Minnesota Medicaid). ${ }^{15}$ Today, MTM services are paid directly and indirectly through a combination of mechanisms including shared-savings arrangements with insurers, ACO contracts with commercial or government payers, or directly via fee-for-service contracts. In a share savings or ACO contract scenario, the system may receive payment for meeting quality or total cost of care benchmarks; the savings paid back to the system can then be used to offset the cost of the MTM pharmacists.

Previously, the program helped improve outcomes and prevent readmissions, for example, but that did not directly yield economic benefits in the fee-for-service system. When a health care system is at risk, the value proposition changes. There is a balance between gaining the revenue from a hospitalization versus being responsible for the total cost of care of a population and therefore wanting to now avoid the hospitalization. Savings that now accrue from interventions such as MTM services that have been shown to improve outcomes and reduce health care costs are even more of a value in the sense that the payment for services is now covered by the savings gained. For example, while the MTM services provided may incur a cost to the ACO, the improved scores on quality metrics would result in bonus payments that potentially cover the cost and increase revenue.

As payment incentives have become better aligned, Fairview is taking a more proactive approach to patient identification than under the fee-for-service system. Historically, patients were referred to the program by physicians. As the ACO has been created and direct employer agreements have been signed, there has been increased use of data and analytics to identify patients who could benefit from the program, most notably from gaps in care data, utilization management, and risk scoring data. The system has built a population data analytics tool to identify patients who are the best fit for each team member based on the data. That team member takes the lead in reaching out and providing care for that patient. Other team members are pulled in as needed. For example, the analytics tool may identify a patient who is not meeting multiple goals of therapy (not meeting hemoglobin Alc targets or blood pressure goals and is not on a statin). That person would be identified as a good MTM candidate. The clinic team would reach out to the patient and schedule an MTM visit.

Another area where aligned incentives are influencing the program is location of MTM pharmacists. In the beginning of the MTM program, Fairview sent a pharmacist to whichever clinic "raised its hand." Learning from past experience, Fairview now takes a more nuanced approached toward deploying MTM pharmacists, since it now better understands its clinic populations, where care gaps might exist, and where these services are needed. 


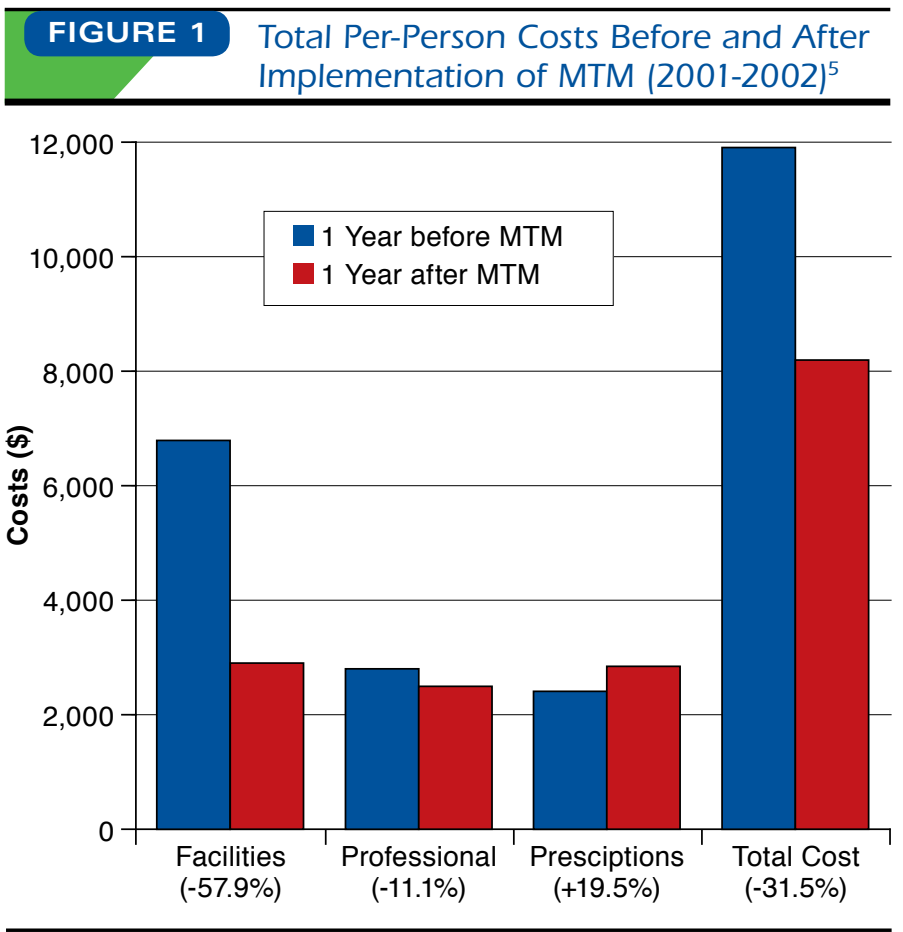

MTM= medication therapy management.

\section{Observations}

Previously conducted studies have confirmed that Fairview's MTM program is improving care and saving money, $5,7,8,16,17$ Since it began, pharmacists have cared for more than 20,000 patients and resolved more than 107,000 medication-related problems, which, if left unresolved, could lead to hospital readmissions and emergency visits. According to Fairview's 10 -year study, about half of the 13,325 patients receiving MTM services from January 1999 through December 2011 had 3 or more drug therapy problems, and one-third had at least 5 problems.${ }^{16}$ Approximately $27 \%$ of the patients needed additional drug therapy, and medication dosages increased. Thirteen percent of the drug therapy problems were the result of unnecessary drug therapy and inappropriately high dosages. ${ }^{16}$ When looking at our Pioneer ACO population-from October 1, 2012, to June 30, 2014-the MTM pharmacists have cared for the highest-risk members and have seen over 670 ACO patients, resolving over 2,780 medication-related problems. The most common types of drug therapy problems addressed are that the dose is too low or that the patient needs additional drug therapy.

In terms of clinical outcomes, the Fairview MTM program published a study showing that these services contributed to optimal care in complex patients with diabetes. ${ }^{17}$ This research compared 121 patients receiving MTM and 103 patients in a control group. The percentage of diabetes patients optimally managed (as measured by a composite of Alc, low-density lipoproteins, blood pressure, aspirin use, and no smoking) was significantly higher for MTM patients (21\% vs. $45 \%) .{ }^{17}$ The MTM program also tracks and reports other clinical markers such as blood pressure, cholesterol, and asthma. These measures are not typically compared with a control group but instead are biannual reported measures. An internal review showed that in the clinical impact of MTM services on patients with asthma, there was a statistically significant change in the Asthma Control Test (ACT) scores from the first MTM visit to the most recent visit: 28 patients (39.5\%) were in range at the first visit, whereas 48 patients $(65.8 \%)$ were in range at the most recent visit. There was a change of $26.3 \%(P<0.001)$.

Patient-centered MTM services can result in positive return on investment (ROI) in both the short and long term. In a study conducted with BlueCross BlueShield of Minnesota from 20012002, Fairview MTM showed a 12:1 ROI when comparing the overall health care costs of patients receiving MTM services when matched to patients who did not receive those services. ${ }^{5}$ Total health expenditures decreased from $\$ 11,965$ to $\$ 8,197$ per person $(\mathrm{n}=186, \mathrm{P}<0.0001)$. The reduction in total annual health expenditures exceeded the cost of providing MTM services by more than 12 to 1 (Figure 1$) .5$ Another study, which examined the outcomes of Fairview's MTM program from September 1998 to September 2008, found that pharmacistestimated cost savings to the health system over the 10-year period were $\$ 2,913,850$ ( $\$ 86$ per encounter), and the total cost of MTM was $\$ 2,258,302$ ( $\$ 67$ per encounter), for an estimated ROI of $\$ 1.29$ for every dollar spent. ${ }^{16}$ At this time, Fairview does not have ROI data for the MTM program for the Pioneer ACO population. This is something that the system would like to analyze, but more time is needed to carry out the interventions as well as receive the claims data for that analysis.

\section{Implications}

There have been important lessons learned that may be helpful to other health care organizations as they develop their own MTM programs. The authors sought additional feedback from other members of The Working Group on Optimizing Medication Therapy in Value-Based Healthcare. This Working Group is composed of pharmacy and health policy leaders from various accountable care organizations, health care performance improvement alliances, and medical group and industry trade organizations who come together to think about key policy issues important for stakeholders involved in accountable care.

The following sections provide insight on how similar programs (Marshfield Clinic in Wisconsin and the Geisinger System in Pennsylvania) operate in their respective ACOs and highlight key implications. 


\section{Costs}

The Fairview MTM program requires a substantial budget to support its pharmacists. The number of pharmacists employed has grown over 15 years, and currently, 18 full-time equivalent pharmacists are employed. For an organization contemplating launching such a program, financial considerations will be among the most critical. How much will it cost to get up and running? What's the timing of the money going out and savings coming in? With how large of a patient population do you start?

There are no simple answers to these questions, and they will likely depend upon the characteristics of each organization. If an organization needs to demonstrate early returns, focusing on acute care admissions/readmissions could be a first step. Thirty-day readmissions have become a focal point for CMS, and the financial penalties associated with them can help justify the MTM program investment. ${ }^{18}$ MTM programs can reduce hospitalizations by targeting newly discharged patients who may be susceptible to rehospitalizations.

Fairview is able to pay for its MTM services directly and indirectly through a variety of mechanisms, including sharedsavings arrangements, ACO contracts, and some direct fee-forservice contracts. Marshfield Clinic in Marshfield, Wisconsin, chose not to bill for MTM services. Leadership there determined that charges on a fee-for-service basis would not generate enough revenue to justify the cost. Instead, Marshfield achieves benefit as part of its value-based care initiatives. When Geisinger Health System in Danville, Pennsylvania, began an anticoagulation program 18 years ago, it also did not charge for the services but aimed for savings from reductions in avoidable complications. Because of the program's success and improved outcomes, Geisinger was subsequently able to sell pharmacist disease management services to payers to generate incremental revenue.

In some environments, patient centered medical homes (PCMHs) receive a per-member-per-month fee to provide population-level and individual patient management. MTM programs provide some of the expected PCMH services, and a portion of the per-member-per-month revenue could compensate for the program costs.

\section{Identifying Patients}

Fairview uses 2 main approaches to identify patients for its MTM program. When it first began, Fairview depended almost exclusively on physician referrals. Now, it also uses analysis of data to target patients who might benefit. These may be patients not meeting their goals of therapy, those missing key drug therapies, or those with high utilization patterns and risk. Marshfield Clinic uses similar methods to identify patients. Through its electronic health record (EHR), Marshfield can identify medications that have been prescribed to its patients. From that information, Marshfield can determine which patients are prime candidates for intervention (e.g., they have diabetes or they have a multiple medication [polypharmacy] issue). In addition to data mining, Marshfield physicians provide an additional source of MTM referrals. Physicians have observed the program's success and actively refer additional patients based upon issues that they observe.

As ACOs take on more clinical and financial responsibility, they need increasingly sophisticated population data analytics. Many ACOs need to move from looking at individual clinic rosters or patient panels to evaluating the system's population. Although it remains important to focus on the patients who come to see them, it is also necessary to shift to those who may not always show up in the clinics. As already stated, an ACO may want to target those not meeting goals, those not on appropriate drug therapy or those on inappropriate drug therapy, and those who are utilizing the emergency services or inpatient setting frequently.

\section{Direct Interaction with Patients}

No single approach to patient interaction will suffice, and appointments do not have to be in person to succeed. Although Fairview's pharmacists are embedded in primary care sites, other methods of interacting with patients are used particularly as new technologies (e.g., telemedicine) have evolved and the imperative to see more patients has grown. Health care systems today are making greater use of telephone appointments as well as taking advantage of a growing variety of Internet options.

At Geisinger, a rural-based integrated delivery system, $40 \%$ of patients are seen face to face by pharmacists. The others are served in a variety of other ways, with the telephone being the most prevalent. Geisinger also uses a webcam through its EHR's patient portal. Although 10\% of Geisinger's patients have no phone, the health system uses other ways to reach them, such as postal mail and messages through neighbors. Marshfield Clinic similarly uses telephone-based "visits" to reach most of its MTM patients because their service area is spread over more than 40,000 square miles.

\section{Role of Protocols for Pharmacists to Change Prescriptions}

There are various approaches to using protocols. At Geisinger and Fairview, pharmacists modify prescriptions based upon approved protocols, and the referring physician is subsequently notified of those changes. Organizations need to be aware that not all states have legislation that allows this practice. In contrast, at Marshfield, pharmacists interact with patients but only make recommendations for medication changes to clinic physicians who finalize those changes. Interestingly, all 3 organizations (Fairview, Marshfield, and Geisinger) believe that what they do enhances team-based care. No one approach is right or wrong; the approach to protocols may have more to do with the culture of the particular organization and the readiness of physicians for independent pharmacist decision making. 


\section{Communication from Pharmacist to Physician}

Once changes to a patient's medication regimen have been made, it is critical that those changes be communicated quickly and efficiently to providers involved in that patient's care. Fairview, Geisinger, and Marshfield Clinic use the EMR and attendant electronic communication channels to inform physicians when medications have been changed. In some situations, the changes are communicated directly from pharmacist to physician because they are both in the same clinic.

\section{Importance of a Visionary Leader}

The insight of a leader has been critical to the Fairview program's success. In 1997, as a result of the new partnership between Fairview and the University of Minnesota, the President of Fairview Pharmacy Services welcomed a collaboration to further develop a new practice model in which pharmacists would be involved in direct patient care and supported that vision throughout the years. He also supported the teaching of students, creating a valuable venue where College of Pharmacy students could gain experience. While other ACO experiences may not be the same as Fairview's, visionaries and leaders come from many places and not always from the executive suite. To be successful, organizations need to identify individuals who can champion the vision and promise of an MTM program.

\section{Limitations}

While the findings in this best practices study may be helpful to many organizations, it may not be possible for all accountable care providers to implement a similar program. As with many aspects of the U.S. health care system, every ACO is unique and must provide services that best fit its patient population. Although the lessons learned by Fairview may help guide other organizations with their MTM efforts, the program will need to be modified and adapted depending on the needs of the ACO.

\section{Recommendations}

The Fairview MTM program described in this article relies on the direct interaction of pharmacists with patients at care sites, while similar programs such as Geisinger's rely more heavily on technologies (e.g., telemedicine) that allow for long-distance consultations. While there is a plethora of literature touting the benefits of in-person and telephonic-based MTM, there is little research to date that directly compares these 2 MTM delivery types. Future research comparing these and other MTM program types may be helpful for ACOs looking to implement or tweak their existing MTM programs to better manage their patient populations.
It will also be critical for research to address direct and indirect costs associated with starting and maintaining an MTM program. Information such as technologies required to start a program and length of time until a program breaks even or meets a sufficient ROI can be helpful for health care providers in similar health systems pitching similar types of programs.

Future research on MTM programs should also investigate the use and dissemination of algorithms, protocols, software programs, or best practices for harnessing, utilizing, and leveraging the richness of electronic clinical data via EHRs. Additional research investigating which data elements provide the best results for minimal cost can be helpful for ACOs looking to effectively best manage their populations.

Although there is already literature examining the role of the pharmacist in direct patient care in a variety of payment and delivery models, there has yet to be significant empirical research into the cost savings of utilizing a pharmacist and MTM services associated with meeting quality and cost benchmarks in an accountable care payment arrangement.

This article has explored Fairview's best practice of a holistic approach to MTM, one mechanism that supports the optimal use of pharmaceuticals in an ACO environment. Other best practices are being developed by the Working Group on Optimizing Medication Therapy in Value-Based Healthcare for complementary issues, such as integrated database analytics, physician readiness for team-based care, role of the pharmacist in an ACO, use of patient-reported measures, and medication disease therapy management for biologics.

\section{Authors}

AMANDA BRUMMEL, PharmD, BCACP, is Director, Clinical Ambulatory Pharmacy Services, Fairview Pharmacy Services, LLC, Minneapolis, Minnesota; MICHAEL A. EVANS, RPh, is Director, Ambulatory Clinical Pharmacy Programs System Therapeutics, Geisinger Health System, Danville, Pennsylvania; GARY S. PLANK, PharmD, was Corporate Director of Pharmacy Services, Marshfield Clinic, Marshfield, Wisconsin; and JERRY PENSO, MD, MBA, is Chief Medical and Quality Officer, American Medical Group Association, Washington DC. ADAM LUSTIG, MS, is Research Manager; KIMBERLY WESTRICH, MA, is Director, Health Services Research; and ROBERT W. DUBOIS, MD, PhD, is Chief Science Officer, National Pharmaceutical Council, Washington, DC.

AUTHOR CORRESPONDENCE: Amanda Brummel, PharmD, BCACP, Director, Clinical Ambulatory Pharmacy Services, Fairview Pharmacy Services, LLC, 711 Kasota Ave. S.E., Minneapolis, MN 55414. Tel.: 612.672.5585; E-mail: arhode1@fairview.org. 


\section{DISCLOSURES}

No outside funding was provided for this research. The authors declare no conflicts of interest. The authors are members of The Working Group on Optimizing Medication Therapy in Value-Based Healthcare. Brummel is employed by Fairview Health Services and has received payment as a speaker from Academy of Managed Care Pharmacy, the American Pharmacists Association, and the American Society of Health-System Pharmacists. Lustig, Westrich, and Dubois are employed by the National Pharmaceutical Council, a health policy research organization that is not involved in lobbying or advocacy. Evans is a member of Merck U.S. Diabetes Board, consults with Roche Diagnostics, and has received payment as a speaker from Janssen Pharmaceutica.

Study concept and design were contributed by Dubois, Westrich, Penso, Brummel, and Lustig. Brummel collected the data, which were interpreted primarily by Brummel, Evans, and Plank, assisted by Dubois and Lustig. The manuscript was written by Lustig, Brummel, Westrich, and Dubois and revised by primarily by Brummel and Lustig, with assistance from Westrich and Dubois.

\section{ACKNOWLEDGMENTS}

The authors thank the other members of The Working Group on Optimizing Medication Therapy in Value-Based Healthcare for their feedback and insight regarding the subject of this article: Julie Day, MD (University of Utah Health Care); Marv Feldman, RPh, MS (Premier); Michael Kelly, MBA (University of Utah Health Care); Gary Kerr, PharmD (Baystate Health); Greg Kotzbauer (Dartmouth); Scott Pope, PharmD (Premier); Albert Rizos, PharmD (Sharp HealthCare); Robert Schoenhaus, PharmD (Sharp HealthCare); and Kimberly D. Westrich, MA (National Pharmaceutical Council).

\section{REFERENCES}

1. Muhlestein D. Why has ACO growth slowed? Health Affairs Blog. October 31, 2013. Available at: http://healthaffairs.org/blog/2013/10/31/why-has-acogrowth-slowed/. Accessed October 30, 2014.

2. Dubois RW, Feldman M, Martin J, Sanderson-Austin J, Westrich KD; Working Group on Optimizing Medication Therapy in Value-Based Healthcare. Role of pharmaceuticals in value-based healthcare: a framework for success. Am J Manag Care. 2012;18(7):e245-47. Available at: http://www.ajmc.com/publications/issue/2012/2012-7-vol18-n7/Role-ofPharmaceuticals-in-Value-Based-Healthcare-A-Framework-for-Success. Accessed October 30, 2014.

3. Dubois RW, Feldman M, Lustig A, et al. Are ACOs ready to be accountable for medication use? J Manag Care Pharm. 2014;20(1):17-21. Available at: http://www.amcp.org/WorkArea/DownloadAsset.aspx?id=17476.

4. American Pharmacists Association. APhA MTM central: what is medication therapy management? 2014. Available at: http://www.pharmacist.com/ $\mathrm{mtm}$. Accessed October 30, 2014.
5. Isetts BJ, Schondelmeyer SW, Artz MB, et al. Clinical and economic outcomes of medication therapy management services: the Minnesota experience. J Am Pharm Assoc (2003). 2008;48(2):203-11.

6. Cipolle RJ, Strand LM, Morley PC. Pharmaceutical Care Practice: The Patient-Centered Approach to Medication. 3rd. ed. New York: McGraw-Hill; 2012.

7. Isetts BJ, Brummel AR, de Oliveira DR, Moen DW. Managing drug-related morbidity and mortality in the patient-centered medical home. Med Care. 2012;50(11):997-1001.

8. Isetts, BJ. Evaluating effectiveness of the Minnesota Medication Therapy Management Care Program: final report. December 14, 2007. Available at: http://archive.leg.state.mn.us/docs/2008/mandated/080113.pdf. Accessed October 30, 2014.

9. Roughead EE, Barratt JD, Ramsay E, et al. The effectiveness of collaborative medicine reviews in delaying time to next hospitalization for patients with heart failure in the practice setting: results of a cohort study. Circ Heart Fail. 2009;2(5):424-28.

10. Nichol A, Downs G. The pharmacist as physician extender in family medicine office practice. J Am Pharm Assoc (2003). 2006;46(1):77-83.

11. Centers for Medicare \& Medicaid Services. Accountable care organizations (ACO). March 22, 2013. Available at: http://www.cms.gov/Medicare/ Medicare-Fee-for-Service-Payment/ACO/. Accessed October 30, 2014.

12. University of Minnesota College of Pharmacy. Pharmacy workup notes. Available at: http://www.pharmacy.umn.edu/medmanagenotes/. Accessed October 30, 2014

13. State of Minnesota. 2014 Minnesota Statutes. Chapter 151.01 Definitions, subd 27: Practice of pharmacy. Available at: https://www.revisor.mn.gov/statutes/?id=151.01. Accessed October 30, 2014.

14. Emerge.MD web site. Available at: www.emergemd.com. Accessed October 30, 2014

15. Minnesota Department of Human Services. Medicaid medication therapy management services. Revised May 1, 2014. Available at: http://www.dhs. state.mn.us/main/idcplg?IdcService=GET_DYNAMIC_CONVERSION\&Revi sionSelectionMethod=LatestReleased\&dDocName=dhs16_136889. Accessed October 30, 2014.

16. Ramalho OD, Brummel AR, Miller DB. Medication therapy management: 10 years experience in a large integrated health care system. J Manag Care Pharm. 2010;16(3):185-95. Available at: http://www.amcp.org/WorkArea/ DownloadAsset. aspx?id=8313.

17. Brummel AR, Soliman AM, Carlson AM, de Oliveira DR. Optimal diabetes care outcomes following face-to-face medication therapy management services. Popul Health Manag. 2013;16(1):28-34.

18. Centers for Medicare $\&$ Medicaid Services. Readmissions reduction program. 2013. Available at: http://www.cms.gov/Medicare/Medicare-Fee-forService-Payment/AcuteInpatientPPS/Readmissions-Reduction-Program.html. Accessed October 30, 2014. 